\title{
Failure of Fermi-liquid theory at strong coupling
}

\author{
V. Janiš \\ Institute of Physics, Academy of Sciences of the Czech Republic, \\ CZ-18040 Praha 8, Czech Republic
}

\begin{abstract}
We investigate quantitatively the effects of strong electron-electron coupling onto the dynamics of lattice electrons. To that purpose the selfconsistent version of the bubble-chain approximation at zero temperature and half filling of the Anderson (Hubbard) model is used. Special attention is paid to a critical region of an electron-hole correlation function shaping the transition from weak to strong interaction. We find an analytic solution with Fermi-liquid properties on the weak-coupling side of the critical region around the two-particle pole. It is shown that Fermi-liquid theory does not lead to a consistent behavior of the self-consistent solution on the strong-coupling side of the critical region.
\end{abstract}

PACS Numbers: $71.27+\mathrm{a}, 71.28+\mathrm{d}$ 
The Anderson and Hubbard models provide a microscopic description of the effects of electron-electron correlations onto the dynamics of a lattice electron gas. Especially recently the single-impurity Anderson model (SIAM) has newly attracted attention of theorists because of its role in the exact description of the Hubbard model in $d=\infty$ [1].2]. However, the two models behave at strong coupling in qualitatively different manner. At half filling and zero temperature, we expect a Kondo-like behavior, i.e. a narrow resonance at the Fermi level, in the SIAM, while the Hubbard model in $d=\infty$, when the antiferromagnetic LRO is suppressed, turns insulating. To understand the differences in behavior of these two related models, it is necessary to have an approximation reliable at intermediate and strong coupling for both Anderson as well as for the Hubbard model.

Although we know much about the SIAM from the Bethe-ansatz solution [3] this method has yet proved inefficient in the Hubbard model in $d=\infty$ in spite of an exact transformation of the $d=\infty$ Hubbard model onto a SIAM with a self-consistent condition. The only technique equally well applicable to the SIAM and to the lattice models is manybody perturbation theory summed via Feynman diagrams.

We know from earlier studies on the SIAM [4, [5] that only self-consistent (renormalized) sums of diagrams can provide reliable approximations at intermediate and strong coupling. Otherwise we cannot evade an unphysical RPA pole in a two-particle Green function [6].

Renormalized sums of Feynman diagrams for the Hubbard model in $d=\infty$ at weak coupling were studied recently [7,8]. It was shown [8] that self-consistent, renormalized sums of the RPA-type can be used at any temperature and in principle at weak as well as at strong coupling. However, it is not straightforward to extrapolate such theories consistently to the strong-coupling regime. There is no analytic solution to these advanced renormalized sums and numerical solutions break down before the strong-coupling limit is reached. The numerical troubles arise when we are approaching the RPA pole (singularity) 
in an electron-hole correlation function. There is then no effective way to make the iterations converge in the strong-coupling regime. It is then crucial to decide from analytic estimates how the pole in the two-particle function is approached by the full, numerically unreachable solution.

The transition from the weak to the strong coupling regime can hence be investigated only analytically using some assumptions. First such an analytic study in the SIAM was done in ref. [9], where a low-frequency approximation was used to estimate the behavior of Suhl's renormalized RPA in the critical region of the two-particle pole. The aim of this paper is to reinvestigate the transition region between weak and strong coupling regimes in the SIAM and the Hubbard model in $d=\infty$. We extend Hamann's approach from ref. [9] based on a Fermi-liquid, low-frequency expansion for electron-hole bubbles and show how dominant contributions to the self-consistent solution can analytically be estimated when the two-particle pole is being approached. As an example we use the self-consistent version of the bubble-chain (shielded interaction) approximation [7,8] applied to the SIAM at half filling and zero temperature. This approximation, in contrast to earlier theories [4, 5.96, represents a thermodynamically consistent and conserving theory [8] applicable to the SIAM as well as to the Hubbard model. As a result we obtain a set of algebraic equations the solution of which forms a Fermi-liquid at weak coupling, but leads to inconsistent results at intermediate and strong couplings. This inconsistency is explained by the failure of the Fermi-liquid, low-frequency ansatz to capture all the relevant features of the full solution at intermediate and strong coupling. It is necessary to take into account also incoherent, non-Fermi-liquid contributions to the two-particle Green function to reach a consistent behavior of diagrammatic approximations at intermediate and strong coupling.

The bubble-chain approximation for the self-energy can generally be written in the spin-polarized version as [7,8] 


$$
\Sigma_{\sigma}\left(i \omega_{n}\right)=-\frac{U^{2}}{2 \beta} \sum_{m=-\infty}^{\infty} G_{\sigma}\left(i \omega_{n}+i \nu_{m}\right) \frac{X_{-\sigma}\left(i \nu_{m}\right)}{1-U^{2} X_{\uparrow}\left(i \nu_{m}\right) X_{\downarrow}\left(i \nu_{m}\right)},
$$

where $X_{\sigma}\left(i \nu_{m}\right)$ is a contribution due to the electron-hole bubble,

$$
X_{\sigma}\left(i \nu_{m}\right)=\frac{1}{\beta} \sum_{n=-\infty}^{\infty} G_{\sigma}\left(i \nu_{m}+i \omega_{n}\right) G_{\sigma}\left(i \omega_{n}\right)
$$

The electron propagator $G_{\sigma}(z)$ is defined for the SIAM as $G(z)=\left[z+\mu_{\sigma}-V^{2} \Gamma_{\sigma}(z)-\right.$ $\left.\Sigma_{\sigma}(z)\right]^{-1}$, where $\mu_{\sigma}=\mu+\sigma h-\epsilon_{f}$ is the effective chemical potential and $\Gamma_{\sigma}(z)$ is the local element of the Green function of the conduction electrons. For the Hubbard model in $d=\infty$ we have $G_{\sigma}(z)=\int d \epsilon \rho(\epsilon)\left[z+\mu-\Sigma_{\sigma}(z)-\epsilon\right]^{-1}$, where $\rho(\epsilon)$ is the density of states (DOS).

We can analytically continue the sums over the Matsubara frequencies $\left(\omega_{n}=(2 n+\right.$ 1) $\pi \beta^{-1}, \nu_{m}=2 m \pi \beta^{-1}$ ) to the real frequencies and after some manipulations we obtain the following representations at $\beta=\infty, n=1$ and $h=0$

$$
\begin{aligned}
& \operatorname{Re} X\left(\omega_{+}\right)=\int_{-\infty}^{0} d x \rho(x) \operatorname{Re}\left[G\left(x+\omega_{+}\right)+G\left(x-\omega_{+}\right)\right], \\
& \operatorname{Im} X\left(\omega_{+}\right)=-\pi \operatorname{sgn} \omega \int_{0}^{|\omega|} d x \rho(x) \rho(x-|\omega|),
\end{aligned}
$$

where $\rho(\omega)=-\frac{1}{\pi} \operatorname{Im} G\left(\omega_{+}\right), \omega_{+}=\omega+i 0^{+}$. The self-energy can then be represented as

$$
\begin{aligned}
\operatorname{Re} \Sigma\left(\omega_{+}\right)= & -\frac{U^{2}}{2} \int_{-\infty}^{0} d x\left\{\rho(x) \operatorname{Re}\left[C\left(x-\omega_{+}\right)-C\left(x+\omega_{+}\right)\right]\right. \\
& \left.+\frac{1}{\pi} \operatorname{Im} C\left(x_{+}\right) \operatorname{Re}\left[G\left(x-\omega_{+}\right)-G\left(x+\omega_{+}\right)\right]\right\}, \\
\operatorname{Im} \Sigma\left(\omega_{+}\right)= & U^{2} \int_{0}^{|\omega|} d x \rho(x-|\omega|) \operatorname{Im} C\left(x_{+}\right) .
\end{aligned}
$$

The two-particle correlation function $C(z):=X(z) /\left(1-U^{2} X(z)^{2}\right)$. Equations (3)-(4) represent a set of nonlinear integral equations for $\operatorname{Re} \Sigma\left(\omega_{+}\right)$and $\operatorname{Im} \Sigma\left(\omega_{+}\right)$. These equations can be solved numerically by iterations at weak coupling [5,7,8], but the iteration 
procedure breaks down as $C(0) \rightarrow \infty$ with increasing $U$. Since $X(0)<0$ the quantity $1+U X(0)$ approaches zero at intermediate coupling. The dominant contributions to $\Sigma(\omega)$ then come from a vicinity of the Fermi energy $(\omega \sim 0)$ where the two-particle correlation function $C(\omega)$ is sharply peaked. We now use the Fermi-liquid assumption that only the low-frequency behavior around the Fermi energy is decisive for the physics of interacting electrons around the two-particle pole and replace the denominator of $C(\omega)$ with a quadratic polynomial

$$
1+U X(\omega) \approx U X^{\prime \prime}(0)\left[\Delta^{2}+\omega^{2}-i \pi a \omega\right]
$$

where $X(0)^{\prime \prime}:=\int_{-\infty}^{0} d x \rho(x) \operatorname{Re} G^{\prime \prime}(x), a:=\nu^{2} / X^{\prime \prime}(0), \Delta^{2}:=(1+U X(0)) / U X^{\prime \prime}(0)$. Here $\nu$ is the DOS of the unpertubed Green function at the Fermi energy. The parameter $\Delta$ is an energy scale measuring dominant fluctuations in the critical region of the two-particle pole. Note that Hamann used in 9] the same idea of a low-frequency expansion at the denominator of a two-particle function, but expanded $X(\omega)$ only to linear power in $\omega$. This difference leads to drastic changes in the critical behavior of the solution. It is also necessary to realize that (5) is valid only if Fermi-liquid theory holds without restrictions, i.e. there are no other relevant energies except for the Fermi one.

Inserting (5) in (丰) we obtain in leading order of the limit $\Delta \rightarrow 0$

$$
\begin{aligned}
& \operatorname{Re} \Sigma\left(\omega_{+}\right)=\operatorname{Re} G(\omega) J(\infty)-\rho(\omega) \operatorname{sgn} \omega K(\omega) \\
& =\frac{a \operatorname{Re} G(\omega)}{2 X^{\prime \prime}(0)} \int_{0}^{\infty} d x \frac{x}{\left(\Delta^{2}+x^{2}\right)^{2}+\pi^{2} a^{2} x^{2}}-\frac{\rho(\omega) \operatorname{sgn} \omega}{2 X^{\prime \prime}(0)} \int_{0}^{|\omega|} d x \frac{\Delta^{2}+x^{2}}{\left(\Delta^{2}+x^{2}\right)^{2}+\pi^{2} a^{2} x^{2}}, \\
& \operatorname{Im} \Sigma\left(\omega_{+}\right)=-\rho(\omega) J(\omega)=-\frac{\pi a \rho(\omega)}{2 X^{\prime \prime}(0)} \int_{0}^{|\omega|} d x \frac{x}{\left(\Delta^{2}+x^{2}\right)^{2}+\pi^{2} a^{2} x^{2}} .
\end{aligned}
$$

We see that integral equations (雨) turned algebraic, where only two positive parameters $\Delta$ and $X^{\prime \prime}(0)$ are expressed as integrals over the products of the full Green function $G(\omega)$. 
The parameter $X^{\prime \prime}(0)$ is proportional to the effective mass $\left(-\Sigma^{\prime}(0)\right)$ of quasiparticles from Fermi-liquid theory and can be assumed as an effective mass of electron-hole pairs. The energy $\Delta$ is a new relevant scale for the two-particle scattering. Although (6) is strictly valid only in the limit $\Delta \rightarrow 0$, we can extrapolate it also to the weak coupling, $U \rightarrow 0, \Delta \rightarrow \infty$. Such a theory then fulfills Fermi-liquid assumtions, i. e. $\operatorname{Im} \Sigma(\omega) \sim-\omega^{2}$ and $\operatorname{Re} \Sigma(\omega) \sim-\omega$ as $\omega \rightarrow 0$, and $\operatorname{Im} \Sigma(\omega) \sim-1 / \omega^{2}$ and $\operatorname{Re} \Sigma(\omega) \sim 1 / \omega$ as $\omega \rightarrow \infty$. Approximation (6) represents the simplest Fermi-liquid theory with frequency dependent self-energy determined essentially from algebraic equations. It may serve as an alternative to the recently proposed approximations trying to clarify the way Fermi liquid breaks down at strong coupling of the Hubbard model [1, 10, 13].

The integrals in (6) can be performed explicitly. To simplify the studied equations we confine our analysis only to the SIAM. If we use the standard approximation $\Gamma\left(\omega_{+}\right)=-i \Gamma$ we can resolve (6) analytically in the limit $\Delta \rightarrow 0$. We find an explicit solution

$$
\begin{aligned}
& \operatorname{Im} \Sigma(\omega)=-\sqrt{\frac{V^{4} \Gamma^{2}}{4}+\frac{J(\omega)}{1+\left(\frac{J(\omega) \omega-\operatorname{sgn} \omega K(\omega) \operatorname{Im} \Sigma\left(\omega_{+}\right)}{J(\omega) V^{2} \Gamma-[J(\omega)+J(\infty)] \operatorname{Im} \Sigma\left(\omega_{+}\right)}\right)^{2}}}+\frac{V^{2} \Gamma}{2}, \\
& \operatorname{Re} \Sigma(\omega)=-\operatorname{Im} \Sigma\left(\omega_{+}\right) \frac{J(\infty) \omega-\operatorname{sgn} \omega K(\omega)\left[V^{2} \Gamma-\operatorname{Im} \Sigma\left(\omega_{+}\right)\right]}{J(\omega) V^{2} \Gamma-[J(\omega)+J(\infty)] \operatorname{Im} \Sigma\left(\omega_{+}\right)} .
\end{aligned}
$$

To close the approximation we complete these equations with definitions of the parameters

$$
\begin{aligned}
X^{\prime \prime}(0) & =\int_{-\infty}^{0} d x \rho(x) \operatorname{Re} G^{\prime \prime}(x) \approx \nu \operatorname{Re} G^{\prime}(0), \\
\Delta^{2} & =\frac{1}{U X^{\prime \prime}(0)}\left[1+2 U \int_{-\infty}^{0} d x \rho(x) \operatorname{Re} G(x)\right] .
\end{aligned}
$$

The set of equations (7) and (8) can be solved numerically. Contrary to Hamann's result we reach a critical interaction $U_{c} \approx 3.7 / \nu$ at which $\Delta=0$ and a pole in the electron-hole 
Green function appears at the Fermi energy. The pole leads, however, to a quite different behavior of the self-consistent solution than in the non-self-consistent RPA. The reason for this behavior deviating qualitatively from that found in RPA on the one side and by Hamann on the other side lies in a breakdown of Fermi-liquid theory around $U_{c}$. The expansion coefficient $X^{\prime \prime}(0)$, neglected by Hamann, diverges at the critical point. Namely $\Delta X^{\prime \prime}(0) \rightarrow \alpha_{c}>0$ and the effective mass of quasiparticles becomes infinite. Hence the low-frequency approximation (5) indicates a sharp transition between weak and strong coupling regimes. The existence of a sharp transition is, however, incompatible with a strong-coupling solution, unless the critical point represents a metal-insulator transition, i.e. the DOS at the Fermi energy vanishes with $\Delta \rightarrow 0$. Namely, it is easy to show that the solution at and above $U_{c}$ does not possess Fermi-liquid properties and

$$
\operatorname{Re} \Sigma\left(0^{-}\right)=-\operatorname{Re} \Sigma\left(0^{+}\right)>0
$$

and when $|\operatorname{Re} \Sigma(0)|<w$, where $w$ is a half bandwidth, then

$$
\operatorname{Im} \Sigma\left(i 0^{+}\right)<0 .
$$

The analyticity assumption for the expansion (5) hence does not hold any longer.

Analyzing the equations at $U \geq U_{c}$ we find that (5) must be replaced by

$$
1+U X(\omega) \approx U X^{\prime}(0)[\Delta+|\omega|-i \pi a \omega]
$$

reflecting a nonanalyticity of the particle-hole bubble at low frequencies at strong coupling. The weak-coupling and strong-coupling ansatzes (5) and (10), respectively, are evidently incompatible and do not allow a continuous matching. There is no critical point from the strong-coupling side as in Hamann's analysis. We must hence conclude that the above analysis based on (5) is incomplete and does not lead to the actual strong-coupling asymptotics of self-consistent diagrammatic approximations. 
Since the real part of the self-energy experiences a jump and the imaginary part acquires a nonzero value at the critical point, expansion (5) around $\omega=0$ in this form becomes meaningless. The frequency interval within which Fermi-liquid theory holds has shrunk to zero at $U_{c}$. To assess the solution in the critical region, $U \nearrow U_{c}$, more precisely we have to take into account two contributions to the function $X\left(\omega_{+}\right)$. The first one is that from (5) and holds now only for $|\omega|<\Theta$. Energy $\Theta$ determines the interval within which Fermi-liquid theory holds. It can be defined as a frequency where the real (imaginary) part of the self-energy reaches its (first) extremum. It is essential that $\Theta \rightarrow 0$ as $\Delta \rightarrow 0$. Function $X\left(\omega_{+}\right)$outside the interval $[-\Theta, \Theta]$ must be newly approximated and one has to expand around the points $\pm \Theta$. Such an expansion has its leading terms of type (10). In the limit $\Delta \rightarrow 0$ the non-Fermi-liquid contributions from the additive expansion around $\Theta \rightarrow 0$ more and more take over the control of the critical behavior and preclude the critical point to be reached. It means that the mechanism how the RPA pole is circumvented in self-consistent approximations with two-particle bubbles is much more complicated than anticipated in Hamann's low-energy analysis within Fermiliquid theory. Only the full treatment of self-consistent diagrammatic approximations with the non-Fermi-liquid contributions (10) can produce the genuine strong-coupling asymptotics. It is not yet clear whether the expected strong-coupling Kondo asymptotics in the SIAM will be reproduced correctly in this way. The result depends namely on a detailed balance of the Fermi- and non-Fermi-liquid contributions in the critical region and on the dispersion relation of the underlying lattice. The complete analysis of the bubble-chain approximation in the critical region $\Delta \rightarrow 0$ will be presented elsewhere.

To conclude, we demonstrated that self-consistent diagrammatic approximations at intermediate and strong coupling, where a pole in a particle-hole correlation function is approached, show interesting behavior going beyond Fermi-liquid theory. We analyzed the 
self-consistent version of the bubble-chain approximation for the Anderson and Hubbard models and proposed a simple analytic solution with Fermi-liquid properties at weak coupling. However, to obtain the asymptotics at strong coupling, it is necessary to restrict the validity of Fermi liquid theory to an interval around the Fermi energy, $\omega \in[-\Theta, \Theta]$, vanishing when the critical point is reached. The contributions obtained from Fermi liquid theory alone are insufficient to suppress the two-particle singularity and to reflect the strong-coupling behavior. Differences in solutions of (6) and that of Hamann from ref. [9] show how a delicate problem it is to find the pertinent asymptotics of the Anderson and Hubbard models in the critical region of a two-particle pole crucial for the transition from the weak to the strong coupling. Neither of the above analytic solutions can yet be seen as exact at strong coupling within the chosen self-consistent approximations.

The work was supported in part by the grant No. 202/95/0008 of the Grant Agency of the Czech Republic. 


\section{REFERENCES}

[1] A. Georges and G. Kotliar, Phys. Rev. B45, 6479 (1992)

[2] V. Janiš and D. Vollhardt, Phys. Rev. B46, 15712 (1992)

[3] T. Kasuya and T. Saso, (eds.), Theory of Heavy Fermions and Valence Fluctuations, (Berlin: Springer 1985)

[4] H. Suhl, Phys. Rev. Lett. 19, 442 (1967)

[5] M. Levine and H. Suhl, Phys. Rev. 171, 567 (1968)

[6] J. R. Schrieffer and D. C. Mattis, Phys. Rev. 140, A1412 (1965)

[7] B. Menge and E. Müller-Hartmann, Z. Physik B82, 237 (1991)

[8] V. Janiš and J. Schlipf, preprint, cond-matt/9507035, to appear in Phys. Rev. B

[9] D. R. Hamann, Phys. Rev. 186, 549 (1969)

[10] X. Y. Zhang, M. J. Rozenberg, and G. Kotliar, Phys. Rev. Lett. 70, 1666 (1993)

[11] A. Georges and W. Krauth, Phys. Rev. B48, 7167 (1993)

[12] D. M. Edwards and J. A. Hertz, Physica B163, 527 (1990)

[13] S. Wermbter and G. Czycholl, J. Phys. C: Condens. Matter 6, 5439 (1994) 